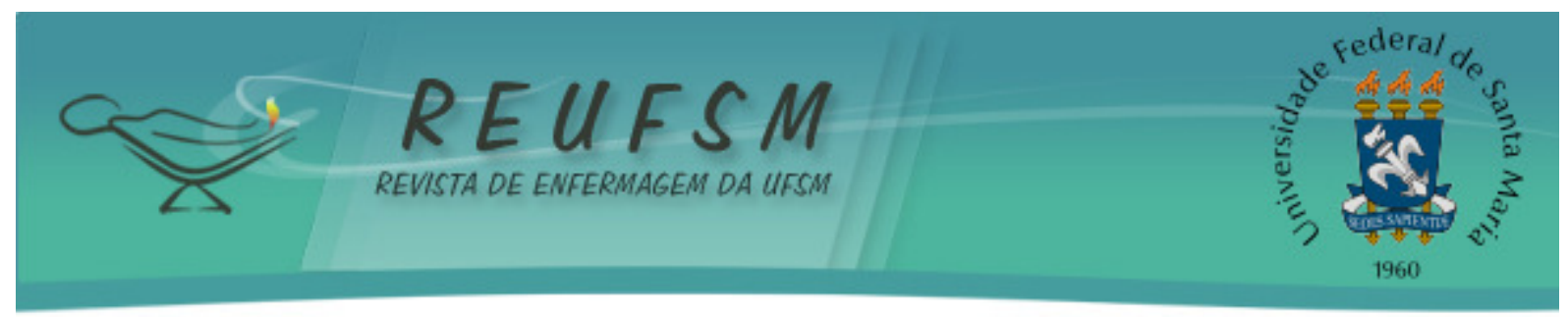

ARTIGO ORIGINAL

\title{
PERFIL DE PACIENTES COM CRISE HIPERTENSIVA ATENDIDOS EM UM PRONTO SOCORRO NO SUL DO BRASIL
}

\section{PATIENTS WITH HYPERTENSIVE CRISIS PROFILE ATTENDED IN A EMERGENCY ROOM IN SOUTHERN BRAZIL}

\section{PERFIL DE PACIENTES CON CRISIS DE HIPERTENSIÓN ATENDIDOS EN UN SERVICIO DE URGENCIA EN EL SUR DE BRASIL}

\author{
Diego Silveira Siqueira ${ }^{1}$ \\ Fernando Riegel ${ }^{2}$ \\ Maria da Graça de Oliveira Crossetti ${ }^{3}$ \\ Juliana Petri Tavares ${ }^{4}$
}

Doi: $10.5902 / 2179769215316$

RESUMO: Objetivo: caracterizar o perfil dos pacientes com crise hipertensiva atendidos em um hospital de pronto socorro no Sul do Brasil. Método: pesquisa descritiva de abordagem quantitativa, com coleta de dados secundários em boletins de atendimento e cadastro eletrônico de pacientes internados por crise hipertensiva, no período de janeiro a maio de 2013. A análise dos dados foi realizada por estatística descritiva e inferencial. Resultados: a associação entre o estágio da hipertensão e faixa etária aponta que o maior percentual dos sujeitos com idade inferior a 40 anos de idade $(44,6 \%)$ apresentavam estágio I de hipertensão, a faixa etária entre 40 e 59 anos, estágio II (34,6\%), e os com idade igual ou superior a 60 anos, estágio III (48,0\%). Conclusão: este estudo identificou a necessidade de criar novas estratégias para a melhoria da adesão ao tratamento da hipertensão arterial e empoderamento do usuário.

Descritores: Hipertensão; Enfermagem; Emergência.

ABSTRACT: Aim: to characterize the patients' profile with hypertensive crisis attended in a hospital emergency room. Method: quantitative, descriptive research with secondary data collection on service bulletins and electronic register of patients admitted with hypertensive crisis on the period from January to May 2013. Data analysis was performed using descriptive and inferential statistics. Results: the association between the stage of hypertension and age shows that the highest percentage of subjects under 40 years old (44.6\%) had stage I hypertension, ages between 40 and 59 stage II (34.6\%), and those ages higher than 60 years old, stage III (48.0\%). Conclusion: this study identified the need to create new strategies to improve treatment adherence for hypertension and user empowerment.

Descriptors:Hypertension; Nursing; Emergency.

\footnotetext{
${ }^{1}$ Enfermeiro. Mestrando do Programa de Pós- Graduação em Medicina da Pontificia Universidade Católica do Rio Grande do Sul. Porto Alegre, RS, Brasil. E-mail: diegoplaneta@ibest.com.br

${ }^{2}$ Enfermeiro. Mestre do Programa de Pós- Graduação em Educação da Universidade do Vale do Rio dos Sinos (UNISINOS). Porto Alegre, RS, Brasil. E-mail: friegel@hcpa.edu.br

3 Enfermeira. Doutora em Filosofia da Enfermagem do Programa de Pós- Graduação em Enfermagem da Universidade Federal de Santa Catarina (UFSC). Docente Titular da Escola de Enfermagem da Universidade Federal do Rio Grande do Sul. Porto Alegre, RS, Brasil. E-mail: mgcrossetti@gmail.com

${ }^{4}$ Enfermeira. Doutora em Enfermagem. Docente do Curso de Enfermagem do Centro Universitário MetodistaIPA. Porto Alegre, RS, Brasil. E-mail: julianapetri@metodistadosul.edu.br
} 


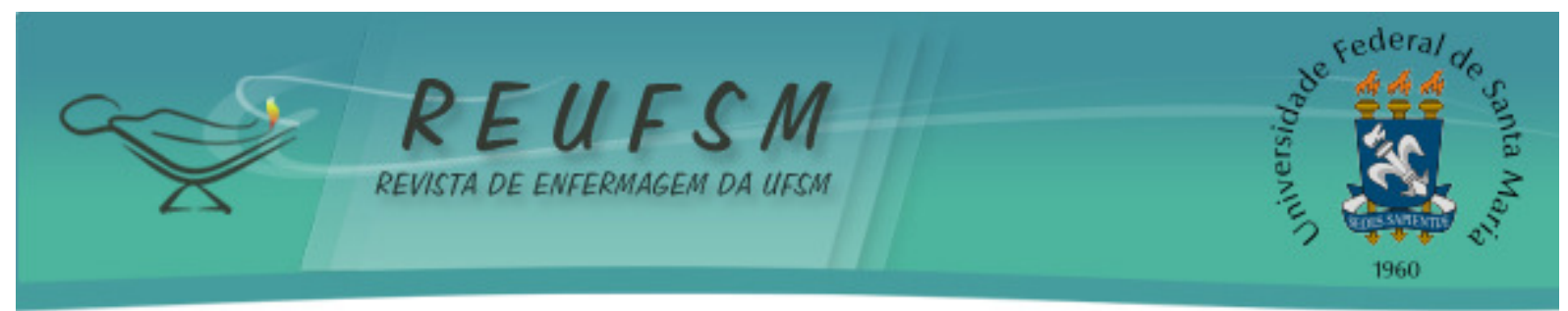

RESUMEN: Objetivos: caracterizar el perfil de los pacientes con crisis de hipertensión con atención en el servicio de emergencia del Sul de Brasil. Método: investigación cuantitativa, descriptiva, con la recopilación de datos secundaria en los boletines de servicio y registro electrónico de pacientes ingresados con crisis hipertensiva en el período entre enero y mayo de 2013. El análisis de los datos ocurrió mediante estadística descriptiva e inferencial. Resultados: la asociación entre la etapa de la hipertensión y la edad muestra que el mayor porcentaje de sujetos menores de 40 años de edad $(44,6 \%)$ presentaban hipertensión en fase I, con edades comprendidas entre 40 y 59 en estadio II (34,6\%), y los menores de 60 años de fase III (48,0\%). Conclusión: el estudio identificó la necesidad de crear nuevas estrategias para mejorar la adherencia al tratamiento de la hipertensión y el empoderamiento del usuario.

Descriptores: Hipertensión; Enfermería; Emergencia.

\section{INTRODUÇÃO}

As doenças crônicas, no Brasil, têm alcançado elevados índices populacionais, fazendo com que o país procure priorizar ações para sua prevenção e controle. Incluída nesse grupo está a hipertensão arterial sistêmica (HAS), considerada um sério problema de saúde pública por acometer $20 \%$ da população adulta mundial. A HAS é uma doença definida pela persistência de níveis tensionais acima dos limites estabelecidos pela Organização Mundial da Saúde (OMS). A hipertensão eleva o risco de acidente vascular encefálico (AVE), doença arterial coronariana (DAC), insuficiência cardíaca (IC), insuficiência renal crônica (IRC) e doença vascular periférica (DVP). ${ }^{1-2}$

A HAS é um importante fator de risco (FR) cardiovascular e torna-se mais preocupante quando associada a outros FR como: obesidade, sedentarismo, tabagismo, dieta inadequada, raça negra, idade avançada e baixo nível socioeconômico. Esse grupo de sujeitos é responsável pela maior procura das emergências e urgências hospitalares. ${ }^{3}$

A crise hipertensiva é uma elevação súbita da pressão arterial, com condições clínicas estáveis, sem comprometimento de órgãos-alvo devendo ser reduzida em 24 horas, muitas vezes com medicamentos via oral. Tal acometimento caracteriza-se pelas condições em que há elevação crítica da pressão arterial com quadro clínico grave, progressiva lesão de órgãos-alvo e risco eminente de morte. Esta condição clínica necessita de uma redução imediata da pressão, devendo ser tratada com agentes anti-hipertensivos parenterais. ${ }^{3}$

Diante deste cenário, este estudo teve por objetivo traçar o perfil dos pacientes e dos atendimentos realizados num Hospital de Pronto Socorro do município de Porto Alegre, Rio Grande do Sul (RS), a fim de traçar estratégias de prevenção, adesão ao tratamento e conscientização da população em relação ao direcionamento dos atendimentos diante de agravos hipertensivos.

\section{MÉTODO}

Trata-se de uma pesquisa de natureza descritiva, com abordagem quantitativa, com coleta de dados secundários em boletim de atendimento e cadastro eletrônico, arquivados no Serviço de Arquivo Médico e Estatístico (SAME). A pesquisa quantitativa envolve a coleta sistemática de informações quantificáveis, mediante condições de extremo controle, além da análise dessa informação com a utilização da Estatística. ${ }^{4}$

A população foi composta pelos boletins de atendimento de pacientes admitidos no setor de emergência do Hospital de Pronto Socorro de Porto Alegre, no período de janeiro a maio de 2013. Foram considerados critérios de inclusão: os prontuários de pacientes que apresentaram diagnóstico de crise hipertensiva no momento da consulta, 


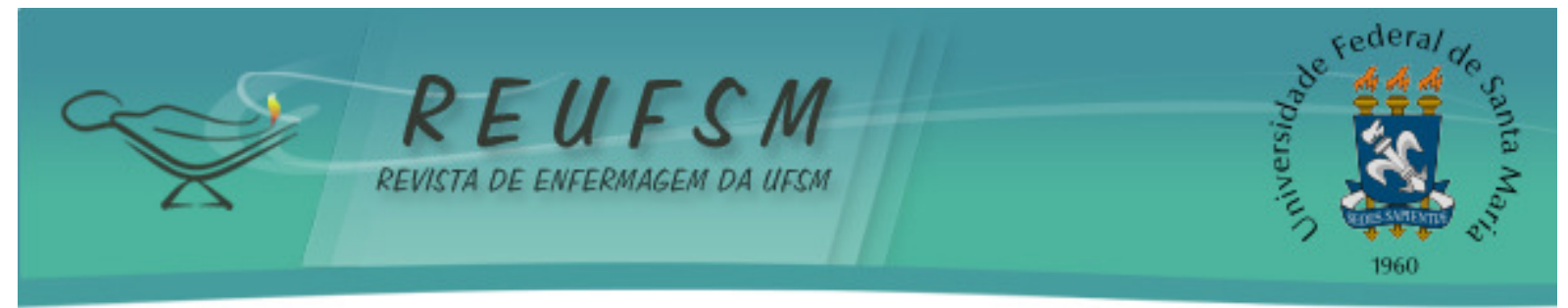

com idade igual ou superior a 18 anos. Para a coleta de dados, foram excluídos os prontuários que não apresentaram informações suficientes, tais como: diagnóstico de crise hipertensiva, queixa principal, tratamento e encaminhamento.

A média de atendimento nesta emergência é de aproximadamente 15.000 atendimentos realizados por mês. Para o estudo proposto, foram necessários, no mínimo 385 prontuários, considerando uma margem de erro de aproximadamente 5 pontos percentuais, um poder de $80 \%$ e nível de significância $p<0,05$.

A coleta de dados foi realizada no Hospital de Pronto Socorro de Porto Alegre - RS, pelo autor através de dados secundários em boletins de atendimento da emergência e cadastro eletrônico, realizado no período de primeiro de novembro de 2013 a 30 de fevereiro de 2014. Esses boletins são disponibilizados no Serviço de Arquivo Medico Estatístico (SAME) do referido hospital. Foi utilizado um instrumento de pesquisa obtendose as seguintes informações: Dados de caracterização da amostra: sexo, data de nascimento, cor, procedência, data do atendimento, escolaridade. Dados clínicos: nível de pressão arterial por estágios, sinais/sintomas e encaminhamento após o atendimento. A amostra deste estudo foi composta por 557 boletins de atendimento do período de janeiro a maio de 2013. Iniciou-se neste período tendo em vista a implementação do protocolo de classificação de risco.

A análise de dados respeitou os critérios referentes à clareza na análise, encadeamento lógico de evidências, construção da explicação e comparação com a literatura científica. ${ }^{5}$

Os dados coletados foram organizados em planilhas no programa Excel $\circledast$, e analisados no programa SPSS ${ }^{\circ}$ (Statistical Package for the Social Sciences, SPSS Inc, Chicago) versão 18.0 para windows.

Os dados foram analisados por meio de técnicas de estatística descritiva e inferencial. As variáveis categóricas estão apresentadas em tabelas, com frequências absolutas e relativas, as variáveis contínuas como medidas de tendência central (média e mediana) e de dispersão (desvio padrão e intervalos quartis).

Para comparar médias entre os grupos com distribuição simétrica, o teste tstudent foi utilizado. Na avaliação de proporções, o teste qui-quadrado de Pearson complementado pela análise dos resíduos ajustados foi aplicado. 0 nível de significância adotado foi de $5 \%(p \leq 0,05)$.

O presente estudo está de acordo com a Resolução 466/2012. ${ }^{6}$ Este projeto foi primeiramente submetido a aprovação pelo Comitê de Ética e Pesquisa do Centro Universitário Metodista do Instituto Porto Alegre (IPA) e posteriormente foi aprovado pelo Comitê de Ética em Pesquisa da Prefeitura de Porto Alegre sob $\mathrm{n}^{\circ}$ de protocolo: 16651813.9.0000.5308.

\section{RESULTADOS}

Em relação aos 557 (100\%) dos boletins de atendimento, evidenciou-se o maior contingente de sujeitos como sendo da faixa etária entre 50 e 59 anos de idade $(24,8 \%)$, seguidos da faixa etária entre 40 e 49 anos (23,5\%), e o menor número de sujeitos correspondeu à faixa etária descrita como maior de 80 anos, correspondendo a um percentual de $2,9 \%$ dos sujeitos. Também foi possível identificar o maior percentual de crise hipertensiva na população feminina (62,5\%). Com relação à raça, prevaleceu à branca como maioria exposta à crise hipertensiva com $75,9 \%$ dos atendimentos, seguidos da raça negra $(12,9 \%)$ e raça parda $(10,6 \%)$. No que tange aos pacientes atendidos, a maioria $(81 \%)$ eram moradores da cidade de Porto Alegre - RS, o que pode ser observado na Tabela 1 a seguir: 


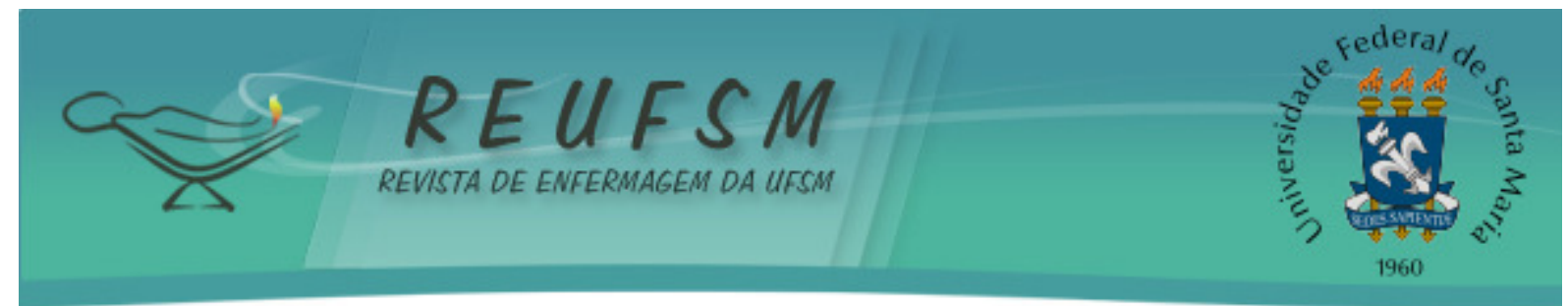

Tabela 1: Caracterização dos pacientes com crise hipertensiva atendidos de janeiro a maio de 2013 no Hospital de Pronto Socorro. Porto Alegre, RS, Brasil, 2014.

\begin{tabular}{|c|c|}
\hline Variáveis & $n=557$ \\
\hline Idade (anos) - média \pm DP & $53,6 \pm 14,4$ \\
\hline Faixa etária & $n(\%)$ \\
\hline$<30$ & $26(4,7)$ \\
\hline $30-39$ & $66(11,8)$ \\
\hline $40-49$ & $131(23,5)$ \\
\hline $50-59$ & $138(24,8)$ \\
\hline $60-69$ & $117(21,0)$ \\
\hline $70-79$ & $63(11,3)$ \\
\hline$\geq 80$ & $16(2,9)$ \\
\hline \multicolumn{2}{|l|}{ Sexo - n(\%) } \\
\hline Masculino & $209(37,5)$ \\
\hline Feminino & $348(62,5)$ \\
\hline \multicolumn{2}{|l|}{ Raça - n(\%) } \\
\hline Branca & $423(75,9)$ \\
\hline Preta & $72(12,9)$ \\
\hline Parda & $59(10,6)$ \\
\hline Indígena & $1(0,2)$ \\
\hline Amarela & $2(0,4)$ \\
\hline \multicolumn{2}{|l|}{ Região - n(\%) } \\
\hline POA & $451(81,0)$ \\
\hline Grande POA & $100(17,9)$ \\
\hline Interior & $6(1,1)$ \\
\hline
\end{tabular}

${ }^{*}$ DP significa desvio padrão

Com relação aos níveis pressóricos encontrados e observados neste estudo, identifica-se maior prevalência de sujeitos com hipertensão em estágio III (40,0\%, n=223), sendo que as mulheres $(n=348)$ apresentam maiores percentuais em relação aos homens $(n=209)$. A média de pressão arterial sistólica foi maior em mulheres $(171,2 \pm 24,0)$ em relação aos homens $(169,7+22,3)$, porém não houve significância estatística $(p>0,05)$ em relação aos dados encontrados na Tabela 2, o que pode ser confirmado a seguir: 


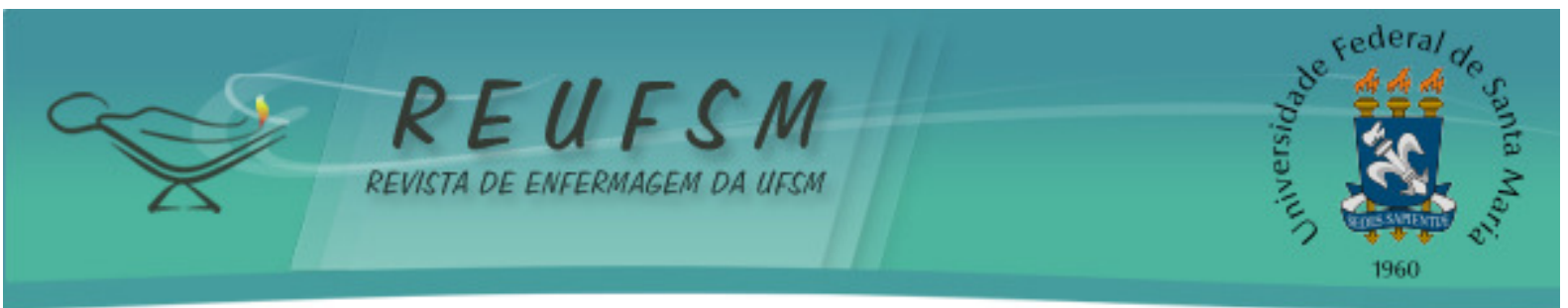

Tabela 2: Dados sobre a pressão arterial dos pacientes atendidos com crise hipertensiva no Hospital de Pronto Socorro de Porto Alegre, RS, Brasil, 2014*

\begin{tabular}{|c|c|c|c|c|}
\hline Variáveis & $\begin{array}{c}\text { Amostra total } \\
(n=557)\end{array}$ & $\begin{array}{l}\text { Homens } \\
(n=209)\end{array}$ & $\begin{array}{c}\text { Mulheres } \\
(n=348)\end{array}$ & $\mathbf{p}$ \\
\hline $\mathrm{PAS}^{*}$ - média $\pm \mathrm{DP} * *$ & $171,2 \pm 23,4$ & $169,7 \pm 22,3$ & $172,1 \pm 24,0$ & 0,256 \\
\hline$P A D^{* * *}$ - média $\pm \mathrm{DP}$ & $106,1 \pm 15,1$ & $106,3 \pm 14,5$ & $106,0 \pm 15,5$ & 0,868 \\
\hline Estágios da Hipertensão - n(\%) & & & & 0,382 \\
\hline 1 & $156(28,0)$ & $57(27,3)$ & $99(28,4)$ & \\
\hline II & $178(32,0)$ & $74(35,4)$ & $104(29,9)$ & \\
\hline III & $223(40,0)$ & $78(37,3)$ & $145(41,7)$ & \\
\hline
\end{tabular}

Em contra partida, a associação entre grau da hipertensão e faixa etária aponta que o maior percentual dos sujeitos com idade inferior a 40 anos $(44,6 \%)$ apresentavam estágio I de hipertensão, os da faixa etária entre 40 e 59 anos de idade, estágio II $(34,6 \%)$, e os com idade igual ou superior a 60 anos apresentavam estágio III $(48,0 \%)$, evidenciando diferença estatística entre os grupos avaliados $(p<0,05)($ Gráfico 1$)$.

Gráfico 1: Associação entre grau da hipertensão e faixa etária dos pacientes com crise hipertensiva atendidos de janeiro a maio de 2013 no Hospital de Pronto Socorro. Porto Alegre, RS, Brasil, 2014.

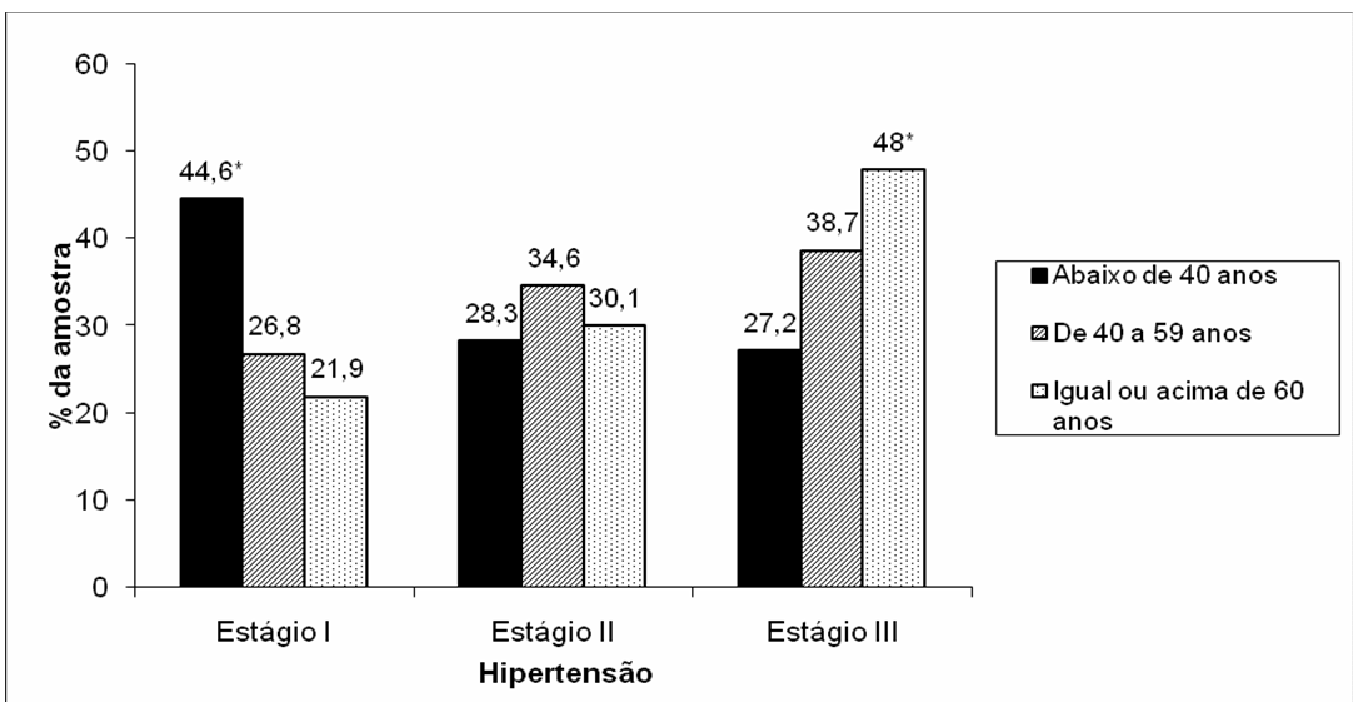

* Associação estatisticamente significativa pelo teste dos resíduos ajustados a 5\% de significância

No Gráfico 2 encontram-se as queixas relatadas pelos pacientes atendidos no Serviço de Emergência do Hospital de Pronto socorro de Porto Alegre. A cefaléia foi a queixa com maior percentual, o que correspondeu a $71,1 \% \quad(n=396)$ dos sujeitos pesquisados, seguida de tontura com $28,0 \%(n=156)$, dor precordial em $17,1 \%(n=95)$ dos sujeitos, e o menor percentual foi dor na nuca com $2,7 \%(n=15)$. 


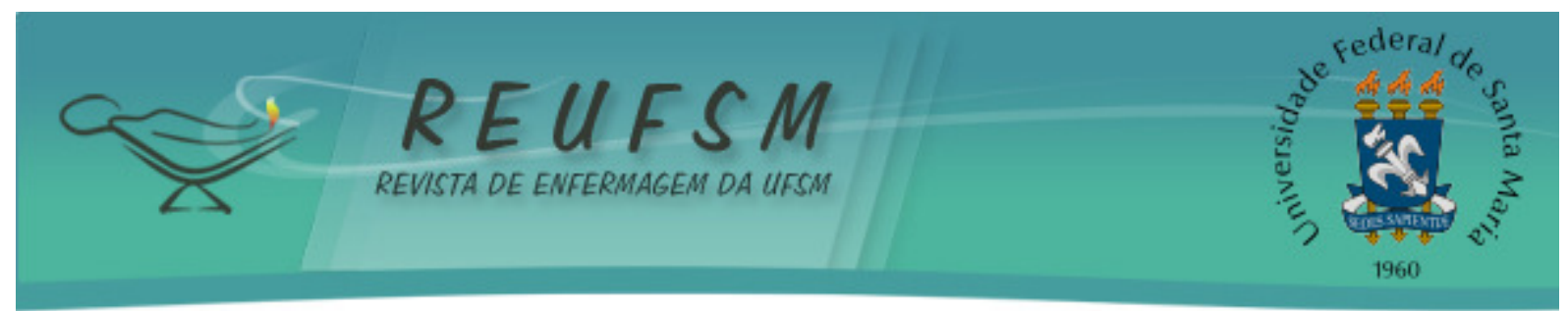

Gráfico 2: Distribuição da amostra quanto às queixas relatadas pelos pacientes com crise hipertensiva atendidos de janeiro a maio de 2013 no Hospital de Pronto Socorro. Porto Alegre, RS, Brasil, 2014.

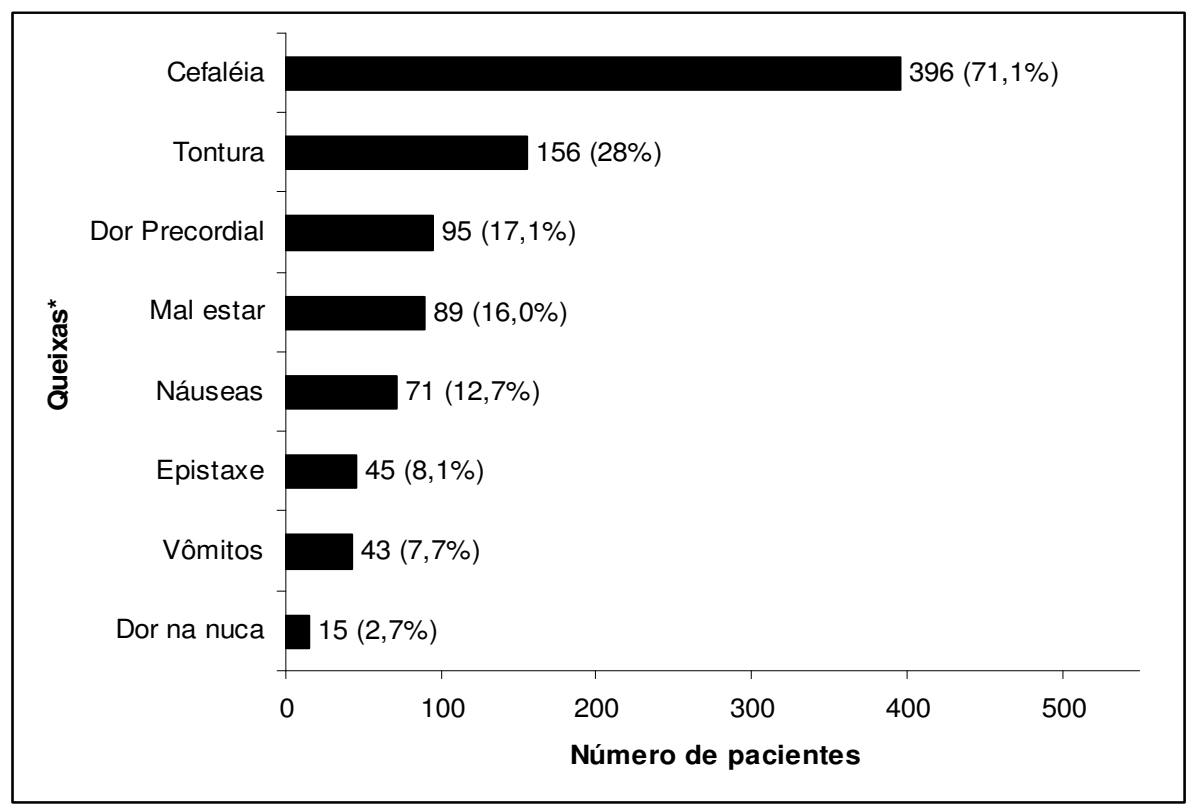

Ainda foi possível identificar que das condutas tomadas a administração de captopril comprimido via oral ou sublingual, presente em $67,0 \%$ dos atendimentos, foi a conduta de maior frequência indicada pela equipe médica. Na sequência a realização do eletrocardiograma em $17,8 \%$ dos antendimentos, importante exame para identificação de alterações eletrocardiográficas também relacionadas com a elevação abrupta dos níveis pressóricos de encontro, como o que é preconizado para atendimento da crise hipertensiva, seguido da administração de medicações analgésicas, tais como: Dipirona Sódica EV 15,8\% ( $n=88)$ e Paracetamol VO em 9,7\% $(n=54)$, Escopolamina em 3,8\% $(n=21)$ e antiemética: Metoclopramida em 10,8\% $(n=60)$, também foi possível verificar a realização do exame sanguíneo de dosagem de enzimas cardíacas realizado em $8,8 \%$ ( $n=$ 49) dos sujeitos do estudo. 


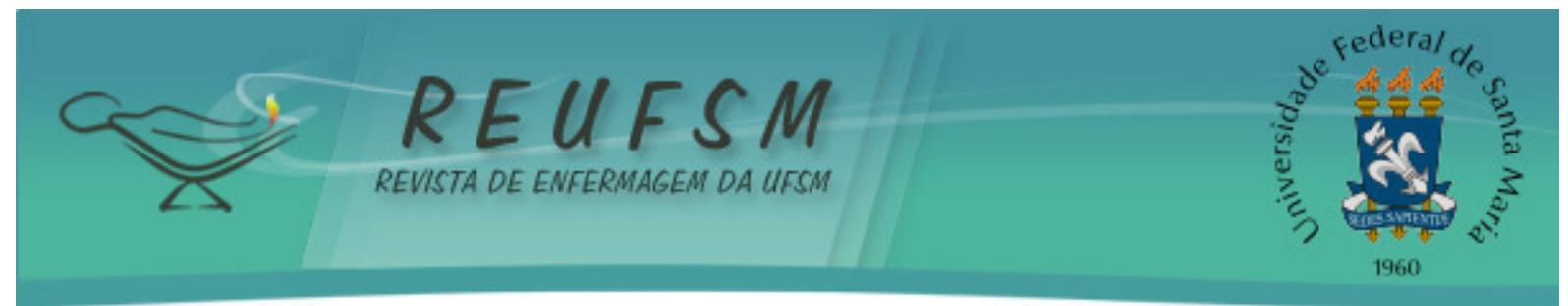

Gráfico 3: Principais condutas adotadas no atendimento dos pacientes com crise hipertensiva atendidos de janeiro a maio de 2013 no Hospital de Pronto Socorro. Porto Alegre, RS, Brasil, 2014.

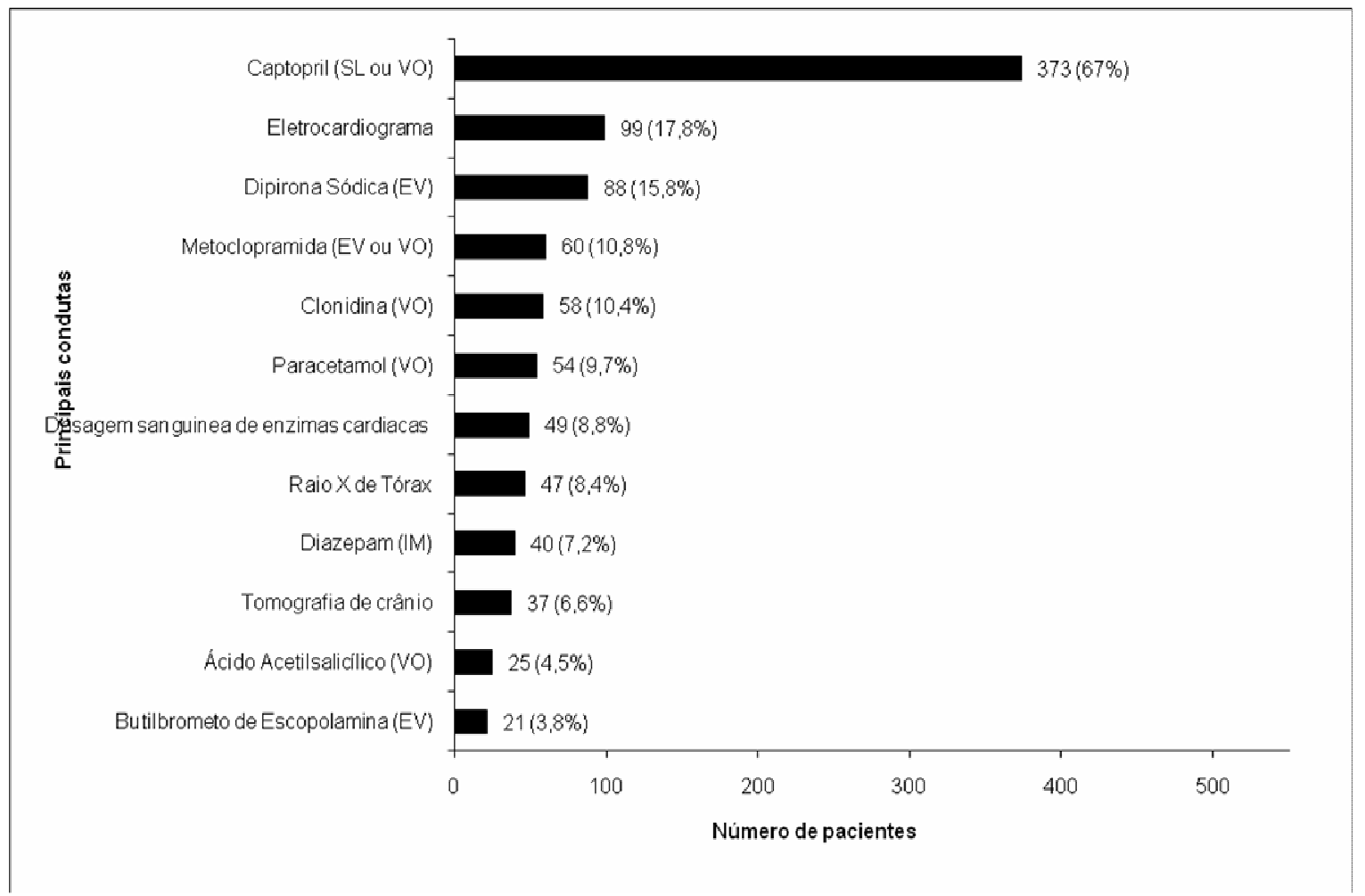

Siglas: SL: via sublingual; VO: via oral; EV: via endovenosa; IM: via intramuscular.

\section{DISCUSSÃO}

0 presente estudo permitiu evidenciar um crescente número de pacientes com estágio I de hipertensão entre jovens, com taxa de prevalência maior entre as mulheres menores de 40 anos de idade.

Pode-se verificar que a maior parte dos pacientes atendidos com crise hipertensiva foi classificada como sendo verde ( $n=298$, o que correspondeu a $53,5 \%$ ), seguidos de amarelo ( $n=245$, equivalente a $44,0 \%$ dos pacientes), azul ( $n=12$, correspondendo a $2,2 \%$ ) e laranja ( $n=2,0,4 \%$ dos atendimentos).

Além disso, considera-se preocupante o fato de hipertensão estágio III não controlada estar acometendo indivíduos com idade entre 40 e 59 anos e igual ou superior a 60 anos, ou seja, mesmo com as facilidades de disponibilização de medicamentos pelo poder público, os pacientes continuam mantendo elevação nos níveis pressóricos. Quais são as possíveis causas? $\mathrm{O}$ problema está na adesão ao tratamento? Na educação em saúde e nas orientações da atenção básica? Na falta de cobertura da Estratégia de Saúde da Família (ESF)? Nos hábitos inadequados de vida que prevalecem mesmo com a ingestão dos medicamentos anti-hipertensivos? Será a falta de adesão da população aos grupos de hipertensos e atividades propostas para prevenção de agravos à saúde? Tantos questionamentos nos remetem a necessidade de avaliar a rigor os achados dessa pesquisa a fim de rever as causas deste desajuste ou melhor, traçar estratégias e rever as políticas públicas deste grupo de pacientes a fim de identificar as intervenções necessárias para o controle da pressão arterial da população estudada. 


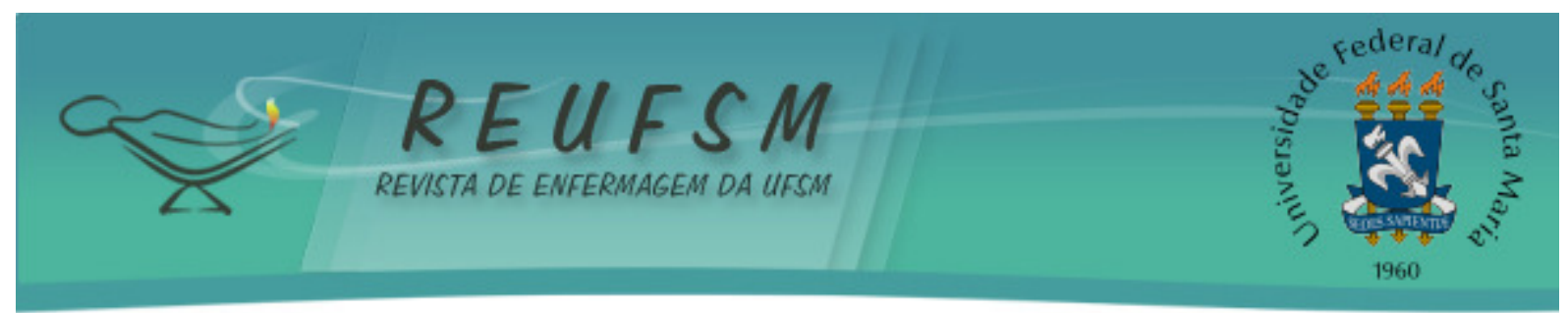

De acordo com a normativa nacional e orientação do Ministério da Saúde (MS) os Serviços de Emergência devem nortear o atendimento de acordo com as Diretrizes preconizadas pela Política Nacional de Atenção às Urgências e Emergências, e neste contexto devem implementar protocolos de classificação de risco para tal. Na Instituição pesquisada utiliza-se como protocolo de Classificação de Risco o Emergency Severity Index (ESI). Frente ao exposto foi possível evidenciar com este estudo como vem sendo classificada a crise hipertensiva e atendidos os pacientes no Serviço de Emergência da Instituição estudada.

Em relação à classificação de risco, esta é tarefa exclusiva do enfermeiro e deve ser realizada por enfermeiros capacitados e com habilidades para reconhecer sinais e sintomas de gravidade, quando da chegada de um paciente em uma unidade de urgência e emergência. Na literatura, há carência de estudos comparando a classificação realizada pelo enfermeiro em relação às cores vermelha, amarela, verde e azul. 0 que foi percebido neste estudo é que os enfermeiros classificaram $53,5 \%$ dos pacientes na cor verde.

0 destino do paciente, após a consulta, foi a alta hospitalar, o que nos faz refletir acerca da baixa gravidade dos atendimentos, apesar da variação dos parâmetros vitais observados, durante a avaliação de risco realizada pelo enfermeiro, situação semelhante vista em estudos nacionais de grandes capitais como São Paulo, Florianópolis e Salvador. ${ }^{7-8}$

Em relação às queixas mais frequentes, entre as relatadas pelos pacientes, estava presente a cefaléia, tontura, dor precordial, mal estar, náuseas, epistaxe, vômitos e dor na nuca. Esses achados são similares aos estudos pesquisados. ${ }^{9-10}$ Chama-nos a atenção para o elevado número de sujeitos sem adesão ao tratamento $(11,1 \% \mathrm{n}=62)$ neste estudo, considerando-se este fato como uma comorbidade por se tratar a hipertensão de um sério problema de saúde pública e a falta de adesão ao tratamento ser uma das razões da elevada taxa de mortalidade.

Em relação aos exames complementares mais solicitados pela equipe médica, neste estudo, exame laboratorial (sangue), raio $x$ de tórax, tomografia de crânio $e$ eletrocardiograma também há abordagem de alguns deles em outros estudo brasileiros, nos quais destacam a importância das unidades básicas demonstrarem ou apresentarem maior resolutividade para a demanda de exames complementares (urina, sangue e eletrocardiograma) serem absorvidos na atenção básica. ${ }^{9-11}$

As condutas tomadas no atendimento dos pacientes que chegam com crise hipertensiva vão de encontro do que preconiza a Sociedade Brasileira de Cardiologia ${ }^{2}$ para tal. Após obtida a redução imediata da pressão arterial, deve-se iniciar a terapia anti-hipertensiva de manutenção e interromper a medicação parenteral. A hidralazina é contra indicada nos casos de síndromes isquêmicas miocárdicas agudas e de dissecção aguda de aorta por induzir ativação simpática, com taquicardia e aumento da pressão de pulso. Em tais situações, indicase o uso de betabloqueadores e de nitroglicerina ou nitroprussiato de sódio. ${ }^{2}$

Em síntese, pode-se inferir que a população está adaptando-se às mudanças no sistema de atendimento do Hospital de Pronto Socorro em relação ao foco que atualmente é o trauma. Percebe-se que ainda procuram o Hospital de Pronto Socorro para resolver casos de crise hipertensiva, porém o sistema de classificação de risco identifica situações que podem ser consideradas graves e com complicações irreversíveis resultado de possível acidente vascular encefálico e encaminha para atendimento médico. As situações em que não há gravidade podem ser encaminhadas para atendimento na rede básica ou unidades de pronto atendimento do município.

Nota-se a necessidade premente de educar a população para a adesão ao tratamento, bem como mudança nos hábitos de vida, além de avaliar os fatores de risco modificáveis para intervenções pontuais de acordo com cada caso. 


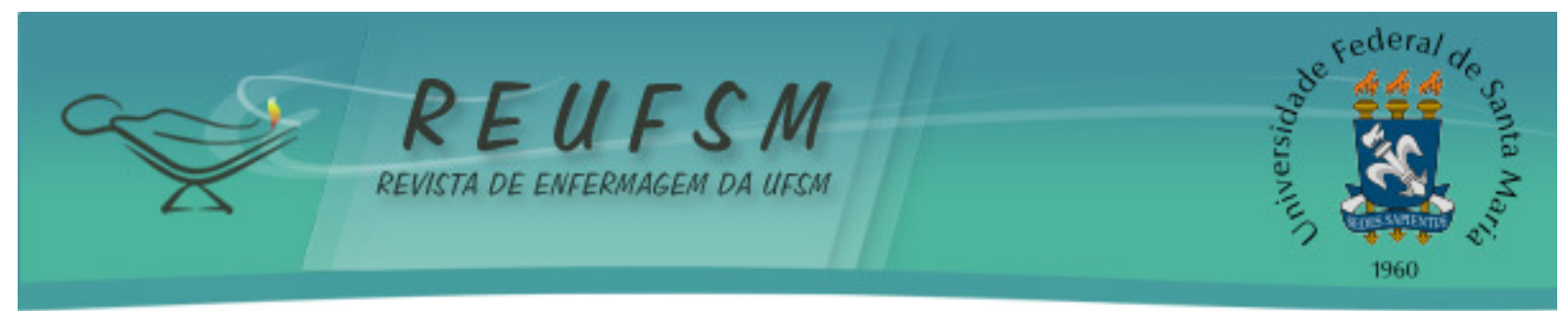

Logo, percebe-se que a população jovem esta ficando hipertensa ao passo que a população idosa não está consciente da necessidade de aderir continuamente ao tratamento, visto que, trata-se de uma doença crônica e sem cura que, na ausência de tratamento, a longo prazo pode prejudicar outros sistemas do organismo desencadeando desajustes e outras comorbidades.

Os achados da pesquisa chamam a atenção para o percentual de $4,7 \%$ dos jovens menores de 30 anos atendidos com crise hipertensiva. Este dado pode estar relacionado ao aumento de hábitos alimentares inadequados, obesidade e sedentarismo na população jovem, corroborando com o estudo que aponta jovens entre 10 e 19 anos assumindo hábitos alimentares prejudiciais à saúde, pois se vêem pressionados por parentes e amigos a investir na aparência e para isso assumem hábitos alimentares não saudáveis ${ }^{11}$

Desse modo, torna-se imprescindível adotar medidas que visem diminuir a superlotação das emergências. Para isso, é fundamental, qualificar a rede de atenção aos pacientes hipertensos, definindo um fluxo assistencial com outros serviços de referência, tais como: unidades básicas de saúde (UBS) e unidades de pronto atendimento (UPA), deixando exclusivamente o pronto socorro para casos como traumas e atendimentos de extrema gravidade. ${ }^{12}$

\section{CONCLUSÃO}

Este estudo evidenciou o perfil dos pacientes e dos atendimentos de crises hipertensivas, trazendo para discussão os subsídios necessários para implementação de ações preventivas e educativas, adesão ao tratamento e controle da pressão arterial.

Dessa forma, foi possível verificar que o maior contingente de sujeitos como sendo da faixa etária entre 50 e 59 anos de idade, do sexo feminino, raça branca e moradores de Porto Alegre. A média de pressão arterial sistólica foi maior em mulheres em relação aos homens, porém não houve diferença estatística entre os grupos. A associação entre grau da hipertensão e faixa etária aponta que maior percentual dos sujeitos com idade inferior a 40 anos de idade apresentavam estágio I de hipertensão, os da faixa etária entre 40 e 59 anos estágio II, e os com idade igual ou superior a 60 anos estágio III. Além disso, a maior parte dos pacientes atendidos com crise hipertensiva foi classificada como sendo verde, apresentavam diagnóstico prévio de HAS, e a principal queixa foi cefaléia.

Observou-se que a maior parte dos pacientes foi encaminhada para UBS sem ser medicado ou receberam qualquer tipo de atendimento, o que poderia colocar a vida do paciente em risco no trajeto do hospital até a UBS. Dentre os eventos que poderiam colocar a vida em risco estão: síncopes, acidente vascular encefálico isquêmico, ou hemorrágico, rompimento de aneurismas, parada cardiorrespiratória, dentre outros. Os pacientes que foram atendidos (medicados) receberam orientações e alta hospitalar, obtiveram o atendimento preconizado pelo MS.

Diante disso, faz-se importante implementar campanhas de prevenção, bem como divulgação dos serviços disponíveis à comunidade no que se refere á informação, como grupos de hipertensos, entre outras atividades como grupos de atividade física, encontros com nutricionistas para elaboração de dietas saudáveis e orientações para a saúde em geral com enfermeiros da atenção básica. Uma vez que os pacientes estão chegando no Pronto Socorro com crise hipertensiva, os serviços de Atenção Primária à Saúde (APS) devem rever suas estratégias de intervenção na atenção à população hipertensa.

Dentre as limitações cabe ressaltar que a realidade do atendimento de emergência é mais complexa em relação aos dados alcançados neste estudo. Também, evidenciou-se que os registros dos boletins de atendimento utilizados neste estudo apresentavam dados muito sucintos e com déficit de dados. Nesse sentido, é fundamental 


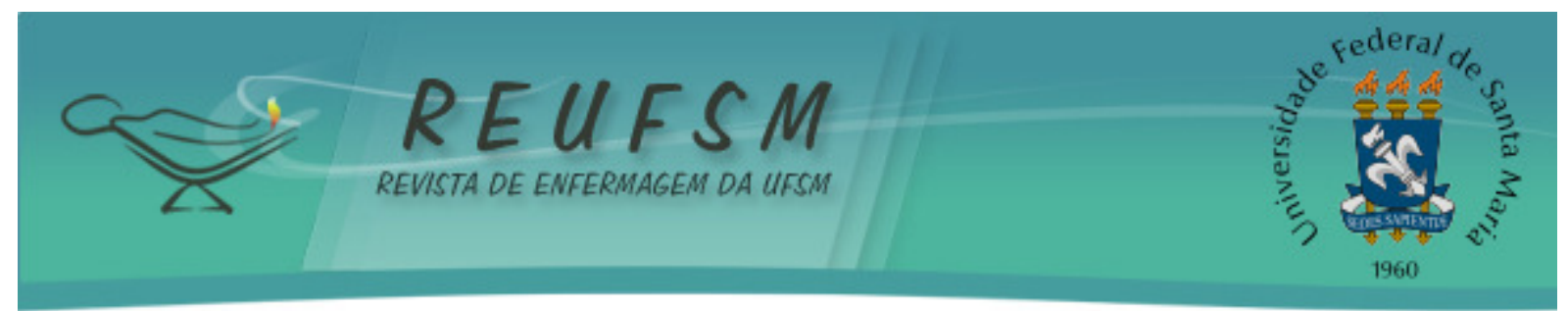

a necessidade premente da implantação do prontuário eletrônico, a fim de unificar as informações dos pacientes juntamente com a capacitação das equipes para o seu preenchimento correto, garantindo o direito dos cidadãos e qualificando o atendimento prestado à população geral.

Por fim, entende-se que a hipertensão arterial é um problema de saúde pública, mas que pode ser controlado. Porém, necessita-se de um Programa abrangente e eficaz de maneira a identificar precocemente os casos existentes, busca ativa da população para a verificação periódica da pressão arterial e a busca por atendimento diante das alterações, aderindo de fato ao tratamento, bem como mudança nos hábitos de vida, prolongando o tempo de vida, minimizando complicações secundárias e agregando qualidade de vida diante do diagnóstico.

\section{REFERÊNCIAS}

1. Carnelosso ML, Barbosa MA, Sousa ALL, Monego ET, Carvalho MM. Enfermidades nãotransmissíveis na atenção básica: novo desafio para o PSF. In: Seclen J, Fernandes AS, organizadores. Experiências e desafios da atenção básica e saúde familiar: caso Brasil. Brasília (DF); 2004. (Série técnica Projeto de desenvolvimento de sistemas e serviços de saúde; 8).

2. Sociedade Brasileira de Hipertensão Arterial (SBHA). VI Diretrizes Brasileiras de Hipertensão Arterial [Internet]. 2010 [acesso em 2015 abr 29];95(1 Supl1):51. Disponível em: http://publicacoes.cardiol.br/consenso/2010/Diretriz_hipertensao_associados.pdf.

3. Martins HS, Brandão Neto RA, Scalabrini Neto A, Velasco IT. Emergências clínicas: abordagem prática. 7a ed. Barueri: São Paulo: Manole; 2012. v. 1. 1086 p.

4. Polit DF, Beck CT. Fundamentos de pesquisa em enfermagem: avaliação de evidências para a prática de enfermagem. $7^{\mathrm{a}}$ ed. Porto Alegre: Artmed; 2011.669 p.

5. Prodanov CC, Freitas EC. Metodologia do trabalho científico: métodos e técnicas da pesquisa e do trabalho acadêmico. $2^{\mathrm{a}}$ ed. Novo Hamburgo: Universidade Feevale; 2013.

6. Brasil. Conselho Nacional de Saúde. Resolução CNS n 466, de 12 de dezembro de 2012. Aprova diretrizes e normas regulamentadoras de pesquisas envolvendo seres humanos [Internet]. 2012 [acesso em 2015 abr 29]. Disponível em: http://conselho.saude.gov.br/resolucoes/2012/Reso466.pdf.

7. Jacobs PC, Matos EP. Estudo exploratório dos atendimentos em unidade de emergência em Salvador - Bahia. Rev Assoc Med Bras [Internet]. 2005 [acesso em 2014 mar 2];51(6):348-53. Disponível

em:

http: / / www.scielo.br/scielo.php?script=sci_arttext\&pid=S0104-42302005000600019.

8. Barakat SFC. Caracterização da demanda do Serviço de Emergências Clínicas de um hospital terciário do município de São Paulo [tese]. São Paulo (SP): Faculdade de Medicina da Universidade de São Paulo; 2001. 120 p.

9. Silva VPM, Silva AK, Heinisch RH, Heinisch LMM. Caracterização do perfil da demanda da emergência de clínica médica do hospital universitário da Universidade Federal de Santa Catarina. Arq Catarin Med [Internet]. 2007 [acesso em 2014 mar 2];36(4):18-25. Disponível em: http://www.acm.org.br/revista/pdf/artigos/520.pdf.

10. Simons DA. Avaliação do perfil da demanda na unidade de emergência em Alagoas a partir da municipalização da saúde e do programa Saúde da Família [tese]. Recife (PE): Fundação Osvaldo Cruz - Centro de Pesquisa Aggeu Magalhães; 2008. 161 p. 


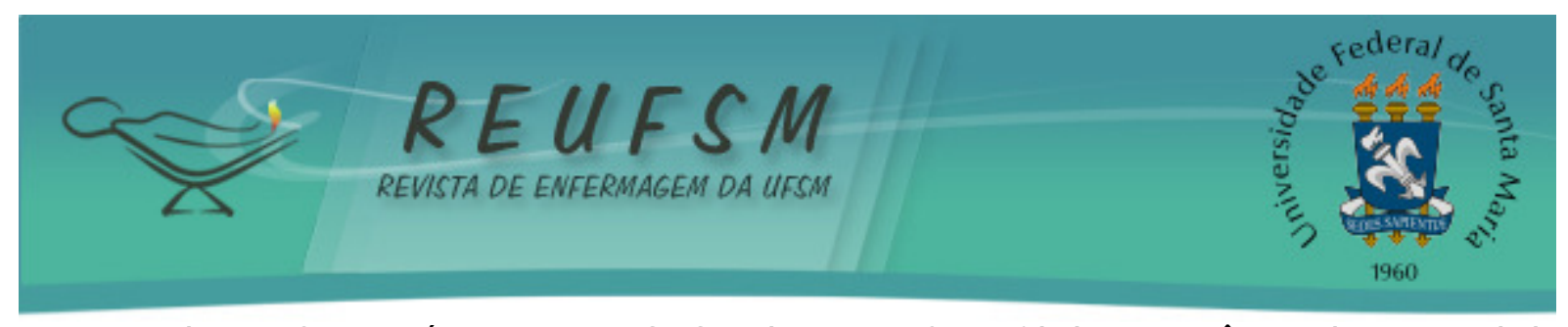

11. Furtado BMASM, Araújo Junior JLC, Cavalcanti P. O perfil da emergência do Hospital da Restauração: uma análise dos possíveis impactos após a municipalização dos serviços de saúde. Rev Bras Epidemiol [Internet]. 2004 [acesso em 2014 mar 23];7(3):279-89. Disponível em: http://www.scielo.br/pdf/rbepid/v7n3/06.pdf.

12. Rosa TP, Magnago TSBS, Tavares JP, Lima SBS, Schimidt MD, Silva RM. Perfil dos pacientes atendidos na sala de emergência do pronto socorro de um hospital universitário. Enferm UFSM [Internet]. 2011 [acesso em 2014 mar 23];1(1): 51-60. Disponível em: http: //cascavel.cpd.ufsm.br/revistas/ojs-

2.2.2/index.php/reufsm/article/view/2090/1511.

Data de recebimento: $27 / 08 / 2014$

Data de aceite: 07/05/2015

Contato do autor responsável: Diego Silveira Siqueira

Endereço postal: Av. Ipiranga, 6690 - Jardim Botânico, CEP: 90610-000, Porto Alegre, RS, Brasil.

E-mail: diegoplaneta@ibest.com.br 